\title{
A unified approach to molecular epidemiology investigations: tools and patterns in California as a case study for endemic shigellosis Sawsan Al-Nimri ${ }^{\dagger 1}$, Woutrina A Miller*†2, Barbara A Byrne², Gerry Guibert ${ }^{3}$ and Lily Chen ${ }^{1}$
}

Address: ${ }^{1}$ Biology Department, San Francisco State University, 1600 Holloway Ave, San Francisco, CA, 94132, USA, ${ }^{2}$ Pathology, Microbiology \& Immunology Department, School of Veterinary Medicine, University of California, Davis, CA, 95616, USA and ${ }^{3}$ Monterey County Public Health Department, 1270 Natividad Rd, Salinas, CA, 93906, USA

Email: Sawsan Al-Nimri - sawsan93940@yahoo.com; Woutrina A Miller* - wamiller@ucdavis.edu; Barbara A Byrne - bbyrne@ucdavis.edu; Gerry Guibert - guibertg@co.monterey.ca.us; Lily Chen - lilychen@sfsu.edu

* Corresponding author †Equal contributors

Published: 24 November 2009

BMC Infectious Diseases 2009, 9:184 doi:10.1186/147|-2334-9-184
Received: 10 March 2009

Accepted: 24 November 2009

This article is available from: http://www.biomedcentral.com/I47I-2334/9//84

(c) 2009 Al-Nimri et al; licensee BioMed Central Ltd.

This is an Open Access article distributed under the terms of the Creative Commons Attribution License (http://creativecommons.org/licenses/by/2.0), which permits unrestricted use, distribution, and reproduction in any medium, provided the original work is properly cited.

\begin{abstract}
Background: Shigellosis causes diarrheal disease in humans from both developed and developing countries, and multi-drug resistance is an emerging problem. The objective of this study is to present a unified approach that can be used to characterize endemic and outbreak patterns of shigellosis using use a suite of epidemiologic and molecular techniques. The approach is applied to a California case study example of endemic shigellosis at the population level.
\end{abstract}

Methods: Epidemiologic patterns were evaluated with respect to demographics, multi-drug resistance, antimicrobial resistance genes, plasmid profiles, and pulsed-field gel electrophoresis (PFGE) fingerprints for the 43 Shigella isolates obtained by the Monterey region health departments over the two year period from 2004-2005.

Results: The traditional epidemiologic as well as molecular epidemiologic findings were consistent with endemic as compared to outbreak shigellosis in this population. A steady low level of cases was observed throughout the study period and high diversity was observed among strains. In contrast to most studies in developed countries, the predominant species was Shigella flexneri (5I\%) followed closely by S. sonnei (49\%). Over $95 \%$ of Shigella isolates were fully resistant to three or more antimicrobial drug subclasses, and $38 \%$ of isolates were resistant to five or more subclasses. More than half of Shigella strains tested carried the tetB, catA, or bla $a_{\text {TEM }}$ genes for antimicrobial resistance to tetracycline, chloramphenicol, and ampicillin, respectively.

Conclusion: This study shows how epidemiologic patterns at the host and bacterial population levels can be used to investigate endemic as compared to outbreak patterns of shigellosis in a community. Information gathered as part of such investigations will be instrumental in identifying emerging antimicrobial resistance, for developing treatment guidelines appropriate for that community, and to provide baseline data with which to compare outbreak strains in the future. 


\section{Background}

Shigellosis, commonly known as acute bacillary dysentery, is a global human health problem, with over 165 million cases and 1 million deaths occurring each year [1]. Shigella sonnei has been reported as the most prevalent endemic species in developed countries, $S$. flexneri as the most prevalent endemic species in developing countries, and $S$. dysenteriae is known to cause sporadic outbreaks and epidemics worldwide [1-5]. In the United States, an estimated 450,000 people are infected each year, with most clinical cases occurring in children $[1,5,6]$.

Although shigellosis is generally a self-limiting disease, antimicrobial treatment can reduce the average duration of symptoms as well as reducing the period of Shigella excretion after symptoms subside $[7,8]$. As a result of antimicrobial use over time, Shigella isolates have developed resistance to many commonly used antimicrobial drugs, and mobile genetic elements including R plasmids, transposons, integrons and genomic islands on the bacterial genome can help disseminate resistance determinants [5,9-11]. Recent reports have determined the molecular basis of multidrug-resistance (MDR) phenotypes of Shigella spp. in Australia [12], Brazil [10], China [13], India [14], Ireland [15], Japan [16], and Korea [17]. Drug resistance patterns are influenced by many factors including geographic location, year in which the isolate was obtained, class of antimicrobial agent, pressure exerted by antimicrobial use, and isolate source $[8,11]$.

Local knowledge of antimicrobial resistance patterns is valuable as a guide to therapy, as an epidemiologic typing method, and as an indicator of disseminating antimicrobial resistance in the region $[11,18]$. Specific PCR assays have been developed to screen for genes associated with virulence and antimicrobial resistance to commonly used drugs such as ampicillin, chloramphenicol, and tetracycline $[5,10,19]$. Plasmid profiling and chromosomal fingerprinting using pulsed-field gel electrophoresis (PFGE) are two molecular techniques that can additionally help to characterize Shigella isolates in endemic and outbreak situations $[15,20]$. Taken together, these methods provide complementary data to compare Shigella strains in a variety of settings and communities.

The objective of this study is to use a suite of epidemiologic and molecular characterization techniques to present a unified approach for characterizing endemic and outbreak patterns of shigellosis with a California case study example at the population level. The results described herein include case distributions, antimicrobial resistance testing, PCR for three antimicrobial resistance genes, plasmid profiling, and chromosomal fingerprinting to evaluate the type of drug resistance, risk factors associated with high levels of drug resistance, and diver- sity of strains present in a central California population during a two year period.

\section{Methods \\ Shigella strains}

Clinical Shigella isolates were obtained from the Monterey and Santa Cruz County Health Departments during 20042005 under the University of California, Davis Institutional Review Board Exemption Protocol 14541-1 for working with anonymous human laboratory samples. The bacteria were inoculated into Selenite-F enrichment broths and onto selective agar plates for isolation and characterization after aerobic incubation at $37^{\circ} \mathrm{C}$ for $24 \mathrm{~h}$. Shigella species were identified using colony morphology and then conventional biochemical reactions including API 20E strips (Biomerieux, France) and antisera slide agglutination tests (BD Difco, MD). Serotype and demographic information was initially provided by the county health departments. The Centers for Disease Control and Prevention reference laboratory performed ipaH PCR and subserotyping of selected Shigella isolates, and this data was used as definitive identification in any case of discrepancies.

\section{Antimicrobial resistance testing}

Drug resistance testing was performed using microdilution and disk diffusion methods as outlined by the Clinical Laboratory Standards Institute [21,22], and antimicrobial classes were defined as for the National Antimicrobial Resistance Monitoring System (NARMS) for enteric bacteria [23]. Sensititre Gram-negative NARMS plates (TREK diagnostic systems, OH) were used to test Shigella isolates for resistance to amikacin (AMK), amoxicillin-clavulanic acid (AMC), ampicillin (AMX), cefoxitin (FOX), ceftiofur (TIO), ceftriaxone (CRO), chloramphenicol (CHL), ciprofloxacin (CIP), gentamicin (GEN), kanamycin (KAN), nalidixic acid (NAL), streptomycin (STR), sulfisoxazole (FIS), tetracycline (TET), and trimethoprim/ sulfamethoxazole (SXT). Inoculated Sensititre ${ }^{\circledast}$ plates were incubated overnight at $35^{\circ} \mathrm{C}$ and examined to determine the dilution at which bacterial growth was at least $90 \%$ inhibited. For quality control, two control strains of Escherichia coli (ATCC 25922 and 35218) were tested as well as the internal positive and negative controls included in each microdilution plate.

Disk diffusion methods were used to test for azithromycin (AZM) resistance [22]. Mueller-Hinton agar plates were inoculated with bacteria suspended in $0.85 \%$ saline adjusted to a turbidity equivalent to a 0.5 McFarland standard and incubated with $15 \mu \mathrm{g}$ azithromycin disks (BBL, BD Diagnostics, NJ) overnight at $37^{\circ} \mathrm{C}$. The area of inhibited bacterial growth around the azithromycin disk was measured to determine whether isolates were resistant $(\leq 13 \mathrm{~mm})$, intermediate $(14-17 \mathrm{~mm})$ or sensitive $(\geq$ 
$18 \mathrm{~mm}$ ). Zone diameters for each isolate were measured manually by two investigators.

\section{PCR analysis}

Selected Shigella isolates were also screened for antimicrobial resistance genes. Fresh colonies were suspended in sterile water, boiled for $10 \mathrm{~min}$, and centrifuged for 10 min at $1300 \mathrm{rpm}$. The supernatant was separated from the pellet and prepared DNA was stored at $-20^{\circ} \mathrm{C}$. Specific primer sets and PCR protocols targeted the tet $B$, cat $A$ and $b l a_{\text {TEM }}$ genes associated with resistance to tetracycline, chloramphenicol, and ampicillin, respectively $[5,10]$. PCR products were run on $1.3 \%$ agarose gels, stained with ethidium bromide, and photographed under UV light on a Gel Doc 2000 system (Bio-Rad, CA).

\section{Plasmid profiling}

Shigella isolates were screened for the presence of plasmid DNA using standard protocols [24]. Plasmid DNA was extracted using an alkaline lysis method with a Quantum Prep Plasmid Miniprep Kit (Bio-rad), $14 \mu \mathrm{l}$ of plasmid DNA extract was added to $4 \mu \mathrm{l}$ of tracking buffer, and the mixture was loaded to a $0.6 \%$ agarose gel. Horizontal gel electrophoresis was carried out in $1 \times$ Tris-acetate-EDTA (TAE) buffer at $80 \mathrm{~V}$ for $1 \mathrm{~h}$. The gel was stained with ethidium bromide and visualized under ultraviolet light (UV) using a Gel Doc 2000 system (Bio-Rad).

\section{PFGE analysis}

Chromosomal fingerprinting using PFGE was performed on selected Shigella isolates according to Pulse Net protocols [25]. Bacteria were incorporated into agarose plugs, lysed, digested with XbaI restriction enzymes (New England Biolabs, MA) and then run on a $1 \%$ gel at $200 \mathrm{~V}$ for $18 \mathrm{~h}$ using a CHEF Mapper system (Bio-Rad). The electrophoresis conditions consisted of an initial switch time of 2.16 seconds and a final switch time of 54.17 seconds. The gel was stained with Gel Star (Cambrex Bioscience, Rockland, ME) and banding patterns photographed under UV light on a Gel Doc 2000 system. Cluster analysis was performed using BioNumerics software (Applied Maths Inc, Austin TX) with comparisons made using Dice coefficients and an unweighted pair-group method with arithmetic averages (UPGMA) [26].

\section{Data analysis}

The distributions of clinical Shigella isolates obtained during the two year study period were grouped by month to evaluate possible endemic versus outbreak patterns in the community. Demographic data provided by the health departments were analyzed with respect to the following categorical variables: Shigella species (S. flexneri, S. sonnei), year of isolation $(2004,2005)$, gender (male, female), and age group ( $<5 \mathrm{yrs}, 5-18 \mathrm{yrs},>18 \mathrm{yrs})$.
Antimicrobial resistance patterns were evaluated with respect to Shigella species, plasmid profiles, and PFGE patterns. Chi-square tests were used to compare categorical groups, and logistic regression was additionally used to evaluate risk factors in relation to the outcome of being resistant to five or more antimicrobial drugs. All analyses were performed using Stata 10.0 software (Statacorp, TX), and $P$-values $<0.1$ were considered significant.

\section{Results \\ Demographics}

During the 2004-2005 study period, 64 Shigella isolates were obtained and the first isolate from each patient was included in this study, resulting in a total sample size of 43 Shigella isolates. Roughly half $(47 \%)$ of isolates were collected in 2004 and 53\% in 2005. The distribution of isolates by month is shown in Figure 1, which is consistent with an endemic level of shigellosis in the community. The highest number of monthly cases was eight in July 2005 and this group was comprised of multiple species, not a single consistent strain that would be suggestive of an outbreak. Overall, the majority (51\%) of Shigella isolated were S. flexneri (serogroup B) while $49 \%$ were S. son$n e i$ (serogroup D). Subserotyping of $S$. flexneri isolates revealed that the most common subserotypes were $3 \mathrm{a}$ (38\% of isolates tested) and $1 \mathrm{~b}(23 \%)$, followed by $1 \mathrm{c}$ and 2a (both $15 \%)$, and lastly a Y variant $(-: 3,4)$ that made up $7 \%$ of $S$. flexneri isolates.

Among Shigella patients, $60 \%$ were female, 36\% were male, and $4 \%$ were of unknown gender. With respect to age, $29 \%$ of Shigella patients were children under 5 yrs, $44 \%$ were $5-18$ yrs, $24 \%$ were over $18 \mathrm{yrs}$, and $2 \%$ were of unknown age. Figure 2 shows the proportion of $S$. flexneri and S. sonnei within each age group. Interestingly, Shigella flexneri was isolated from patients in all age groups, in contrast to $S$. sonnei that was only isolated in patients under 18 yrs.

\section{Antimicrobial Susceptibility}

Antimicrobial drug testing was carried out on 42 Shigella isolates divided evenly among $S$. flexneri and $S$. sonnei. Figure 3 shows the percent of $S$. flexneri and $S$. sonnei isolates fully resistant to individual antimicrobial drugs in 2004 compared to 2005 . Over $60 \%$ of $S$. flexneri isolates were fully resistant to ampicillin, chloramphenicol, or tetracycline in both years. Increased resistance of S. flexneri to amoxicillin-clavulanic acid, kanamycin, streptomycin, sulfizoxazole, and trimethoprim-sulfa was observed in 2005 compared to 2004 . For S. sonnei, over $80 \%$ of isolates in both years were fully resistant to streptomycin, sulfizoxazole, tetracycline, or trimethoprim-sulfa. Less than $50 \%$ of $S$. sonnei were fully resistant to ampicillin, chloramphenicol, or kanamycin. No full drug resistance 


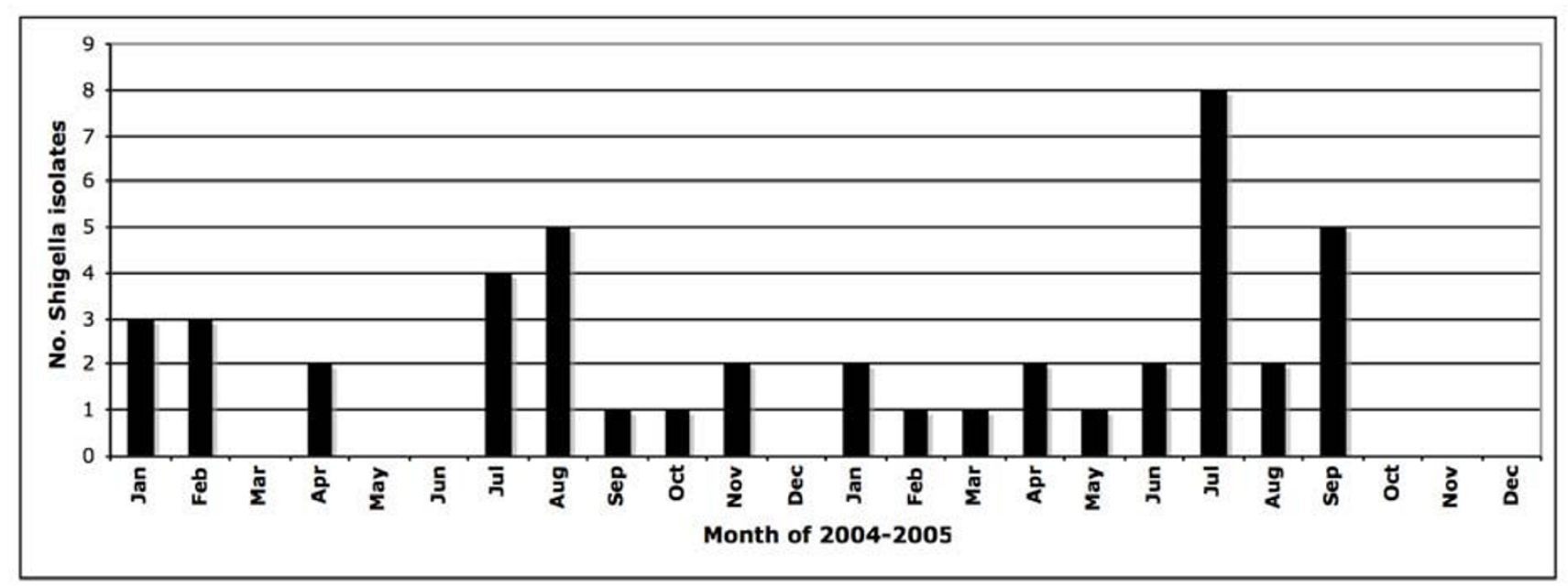

Figure I

Monthly Shigella distribution in the Monterey Bay region of California, 2004-2005.

in Shigella spp. was observed for amikacin, azithromycin, cefoxitime, ceftiofur, ceftriaxone, ciprofloxacin, gentamicin, or nalidixic acid. It is significant to note that while none of the Shigella isolates were fully resistant to azithromycin in this study, $86 \%$ of $S$. sonnei isolates showed intermediate resistance, as did $10 \%$ of $S$. flexneri isolates. Similarly for amoxicillin-clavulanic acid, $48 \%$ of S. flexneri had an intermediate level of resistance, as did $10 \%$ S. sonnei isolates.

In addition to drug resistance to individual agents, multidrug resistance is also important to consider. Over 95\% of

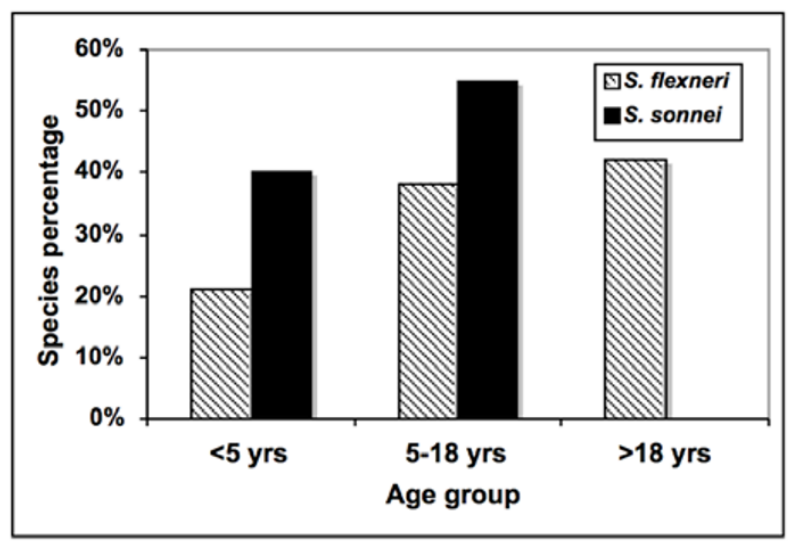

Figure 2

Age distribution of Shigella species from the Monterey Bay region of California, 2004-2005. S. flexneri - Shigella flexneri; S. sonnei - Shigella sonnei.
Shigella isolates in this study were resistant to three or more antimicrobial drug subclasses as defined by NARMS criteria and $38 \%$ of isolates were resistant to five or more. Figure 4 shows the number of drugs that $S$. flexneri and $S$. sonnei isolates were resistant to in 2004 and 2005. The majority of both species were resistant to four or more drugs in both years. The level of multi-drug resistance increased from 2004 to 2005 for S. flexneri isolates but the shift was less dramatic for $S$. sonnei.

Table 1 shows the drug resistance patterns (antibiograms) in this study, listed from most to least common. A third of isolates were fully resistant to streptomycin, sulfisoxazole, tetracycline, and trimethoprim-sulfa. The next most common patterns were to ampicillin, streptomycin, sulfisoxazole, tetracycline, and trimethoprim-sulfa $(14 \%$ of isolates), and then to ampicillin, choramphenicol, streptomycin, sulfosoxazole, and tetracycline (10\% of isolates). The highest level of resistance (six antimicrobial drugs) was detected in $7 \%$ of Shigella isolates and included ampicillin, chloramphenicol, streptomycin, sulfisoxazole, tetracycline, and trimethoprim-sulfa.

Logistic regression analysis identified two risk factors statistically associated with high drug resistance as defined by full resistance to five or more drugs. In the univariate analysis, high drug resistance was more likely to be associated with $S$. flexneri than $S$. sonnei $(P=0.06)$, and more likely to be detected in female than male patients $(P=$ $0.07)$. Age group and year of isolation were not significant risk factors. However, when both Shigella species and patient gender were included in the regression model, $S$. flexneri species was positively associated with high drug resistance $(P=0.09)$, while gender was not significant $(P$ 

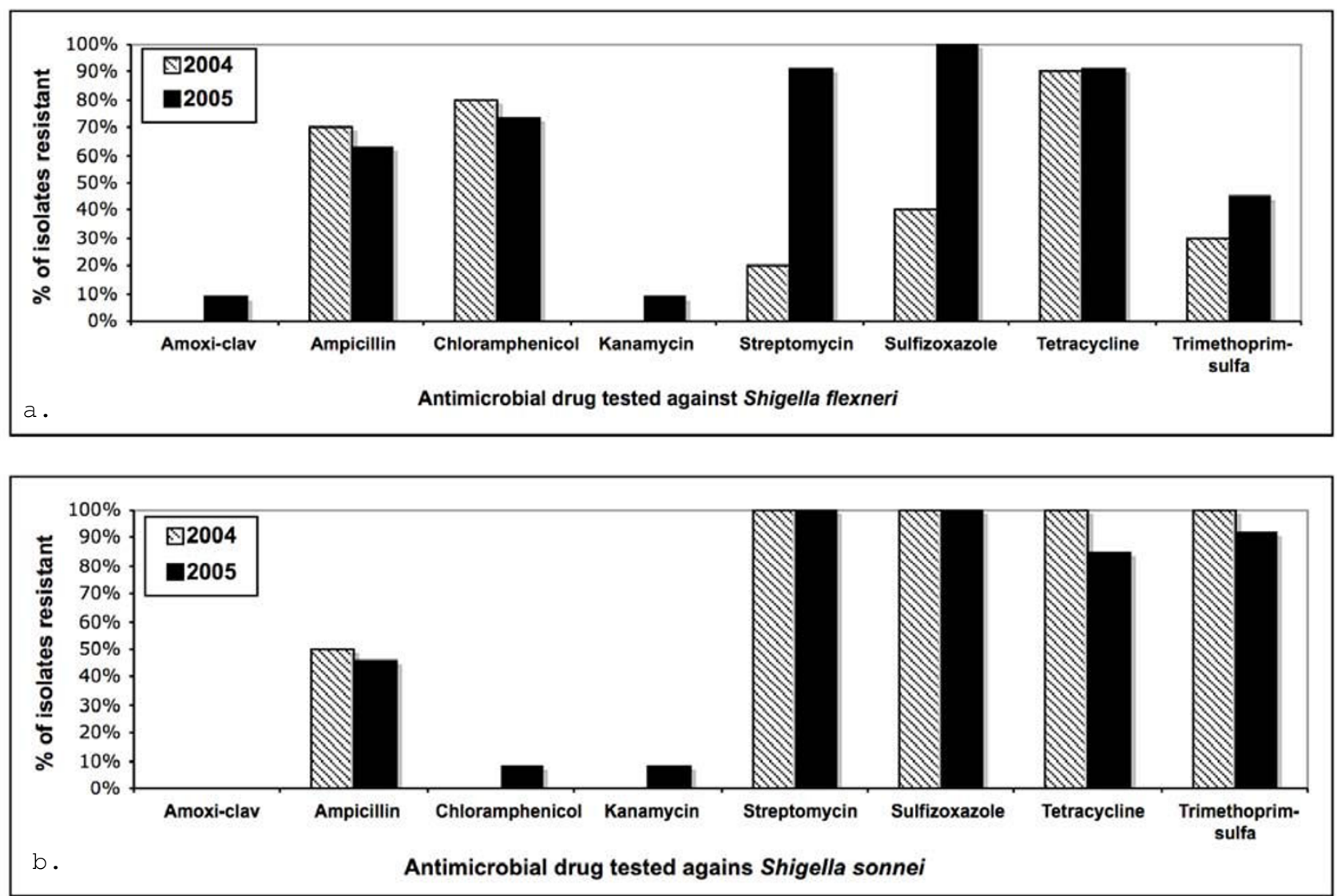

Figure 3

Percent of Shigella flexneri (top) and S. sonnei (bottom) isolates fully resistant to antimicrobial drugs. No full resistance was observed to amikacin, azithromycin, cefoxitime, ceftiofur, ceftriaxone, ciprofloxacin, gentamicin, or nalidixic acid.

$=0.12$ ). In the final model, high drug resistance was 1.2 times more likely in S. flexneri than S. sonnei, and other risk factors were not significant.

\section{PCR analysis}

Molecular PCR amplification resulted in gels with the 415, 457, and 559 bp bands expected for genes associated with tetracycline (tetB), chloramphenicol ( $\operatorname{cat} A)$, and ampicillin $\left(b l a_{\mathrm{TEM}}\right)$ resistance, respectively. Of the 33 tetracycline-resistant Shigella tested, $100 \%(16 / 16)$ of $S$. flexneri and $71 \%$ (12/17) of S. sonnei were positive for the tet $B$ gene. Of the 14 chloramphenicol resistant isolates tested, $85 \%(11 / 13)$ of S. flexneri and 100\% (1/1) S. sonnei were positive for the catA gene. Of the 24 ampicillin resistant Shigella tested, 21\% (3/14) of S. flexneri and 44\% (4/ $9)$ of $S$. sonnei were positive for the $b l a_{T E M}$ gene.

\section{Plasmid profiling}

Ten different plasmid profiles (I-X) were detected in this study, with plasmid bands ranging from $1.5-10 \mathrm{~kb}$ in size. Of the 42 isolates tested, $62 \%$ has plasmids and $38 \%$ did not. Profile I had 1.5 and $2 \mathrm{~kb}$ plasmids, profile II had 1.5, 4 , and $5 \mathrm{~kb}$ plasmids, profile III had $1.5,2,3,5,6$, and 10 $\mathrm{kb}$ plasmids, profile IV had 2 and $2.5 \mathrm{~kb}$ plasmids, profile $\mathrm{V}$ had $1.5,2$, and $3 \mathrm{~kb}$ plasmids, profile VI had 1.5 and 3 $\mathrm{kb}$ plasmids, profile VII had $1.4,2,2,5$, and $3 \mathrm{~kb}$ plasmids, profile VIII had 2 and $4.5 \mathrm{~kb}$ plasmids, profile IX had $1.5,3$, and $4 \mathrm{~kb}$ plasmids, and profile $\mathrm{X}$ had 1.5, 3, 4, and $5 \mathrm{~kb}$ plasmids. Table 1 shows the distribution of plasmid profiles and serotypes by antibiogram group, with some antibiogram groups including multiple plasmid profiles and serotypes. 

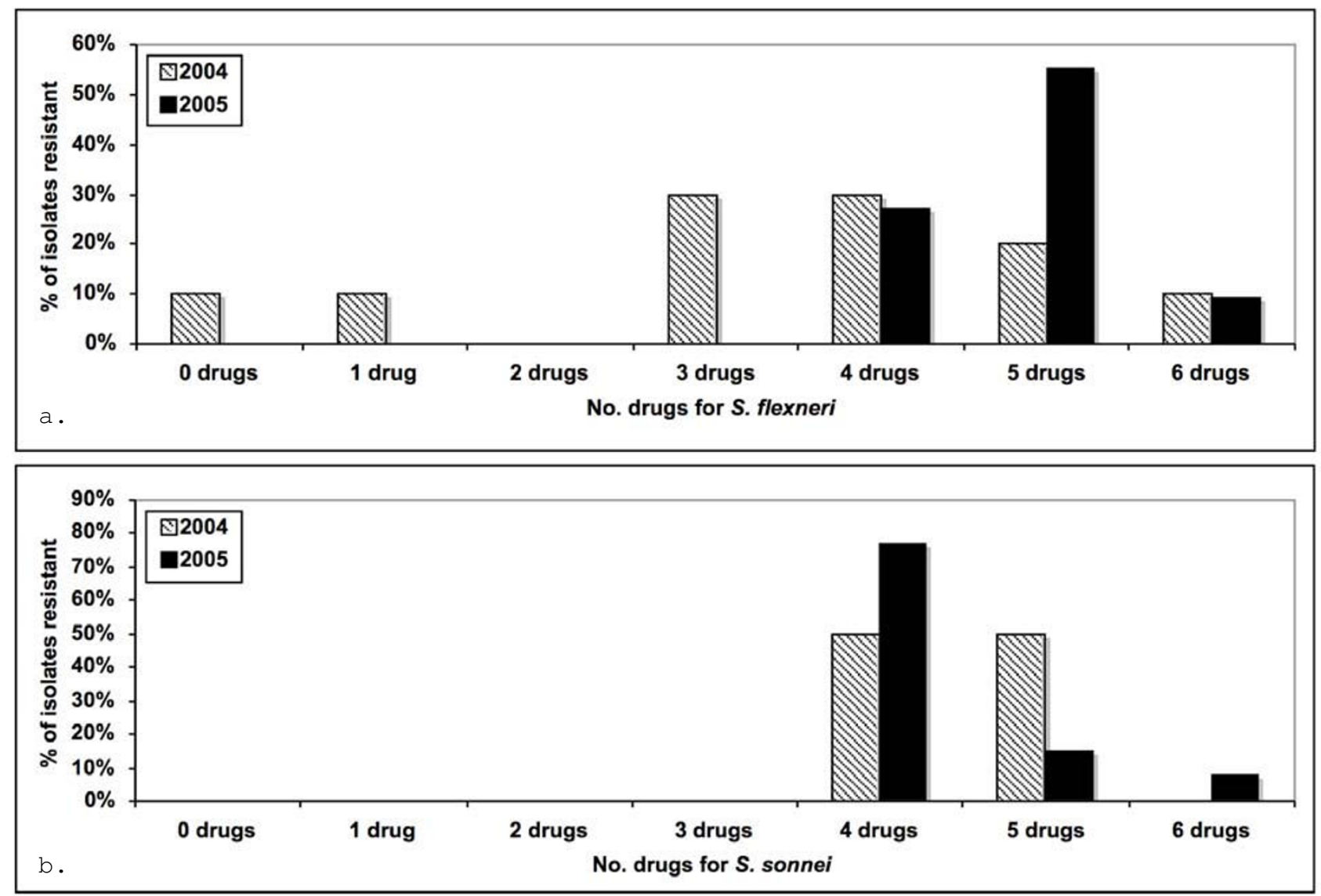

Figure 4

Proportion of multi-drug resistant Shigella flexneri (top) and S. sonnei (bottom) isolates from California, 20042005.

\section{PFGE}

Twenty-six Shigella isolates were selected for PFGE fingerprinting, with an even number of S. sonnei and S. flexneri represented, and at least one isolate from each plasmid profile included in the chromosomal fingerprinting analysis. The PFGE patterns after XbaI digestion are shown in Figure 5 as dendrograms of genetic intra-species relatedness. All PFGE patterns were unique and thus show that there is a high level of diversity in Shigella from the Monterey region of California. This provides molecular evidence to support the endemic epidemiologic pattern in time that was observed in the initial epidemiologic evaluation (Figure 1). The distribution of PFGE patterns by antibiogram group and in relation to plasmid profile groups and serotypes are shown in Table 1, again highlighting the diversity of endemic strains in this population.

\section{Discussion}

This study shows how epidemiologic patterns at the host and bacteria population levels can be used to investigate endemic as compared to outbreak patterns of shigellosis in a community. The observed pattern in time, with a small number of cases coming in throughout the year, along with the high diversity in molecular fingerprints of Shigella isolates support an endemic pattern in this population. A true spatial analysis would also be useful to evaluate clustering and additional risk factors for infection, but due to patient confidentiality that data was not available. Traditional and molecular epidemiologic data gathered as part of public health investigations will be instrumental in identifying emerging antimicrobial resistance, developing treatment guidelines appropriate for communities, and providing baseline data with which to compare future outbreak strains. 
Table I: Shigella drug resistance patterns with associated serotypes, plasmid profiles, and PFGE fingerprint patterns from California, 2004-2005.

\begin{tabular}{|c|c|c|c|c|}
\hline $\begin{array}{l}\text { Resistance pattern to the } \\
\text { NARMS antimicrobial } \\
\text { panel }\end{array}$ & $\begin{array}{l}\% \text { of isolates with } \\
\text { resistance profile (no.) }\end{array}$ & $\begin{array}{l}\text { Plasmid profile groups } \\
\text { detected (no.) }\end{array}$ & $\begin{array}{l}\text { Species of isolates with } \\
\text { plasmids (no.) }\end{array}$ & PFGE patterns \\
\hline STR, FIS, TET, SXT & $33 \%(14)$ & II, X(3), VIII & S. sonnei (4), S. flexneri & III, V, VI, IX, X \\
\hline AMP, STR, FIS, TET, SXT & $14 \%(6)$ & II(4), VI & S. sonnei (5) & I, IV, VII, VIII, XI, XII \\
\hline AMP, CHL, STR, FIS, TET & $10 \%(4)$ & none & none & \\
\hline AMP, CHL, TET & $7 \%(3)$ & VII & S. flexneri 3a & $X X, X X I I I, X V$ \\
\hline AMP, CHL, STR, FIS, TET, SXT & $7 \%(3)$ & $\mathrm{IV}(2), \mathrm{V}$ & S. flexneri 3a (2), S. sonnei & \\
\hline TET & $5 \%(2)$ & I, IV & S. flexneri Ib (2) & XIX \\
\hline AMP, CHL, FIS, TET & $5 \%(2)$ & $\operatorname{IV}(2)$ & S. flexneri $2 \mathrm{a}(2)$ & $X V I I$ \\
\hline CHL, STR, FIS, TET, SXT & $5 \%(2)$ & $\mathrm{V}(2)$ & S. flexneri Ic (2) & $X V$ \\
\hline AMP, CHL, FIS, TET, SXT & $2 \%(I)$ & IV & S. flexneri $2 \mathrm{a}$ & XVIII \\
\hline AMP, KAN, STR, FIS & $2 \%(1)$ & IV & S. sonnei & XIII \\
\hline CHL, FIS, TET, SXT & $2 \%(I)$ & III & S. flexneri Y var. & XXIV \\
\hline AMP, CHL, STR, TET & $2 \%(I)$ & IX & S. flexneri Ib & XIV \\
\hline AMP, STR, FIS, SXT & $2 \%(I)$ & IX & S. sonnei & \\
\hline AMC, AMP, GEN, STR, FIS & $2 \%(I)$ & IV & S. flexneri & \\
\hline
\end{tabular}

a Of the 42 isolates tested, $62 \%(n=26)$ had plasmids detected while $38 \%(n=16)$ did not.

There is worldwide concern about the emergence of drug resistant strains of enteric pathogens. In this study, we found that over $95 \%$ of Shigella isolates from the Monterey region of California were resistant to three or more antimicrobial drug classes, and 38\% were resistant to five or more. This level of drug resistance is higher than national NARMS data for the same 2004 time period, which reported $62 \%$ resistance to three or more drug subclasses, and $28 \%$ resistance to five or more [23].

Another difference between this central California study and national data is that in this study $S$. flexneri was the more prevalent species, followed closely by $S$. sonnei. Most studies have reported $S$. sonnei to be the most common species in developed countries such as the United States, with $S$. flexneri more prevalent in developing countries [1$4,27,28]$. For example, the Shiferaw [28] study reported that $S$. sonnei accounted for $70 \%$ of Shigella infections in the United States, followed by $S$. flexneri associated with $24 \%$ of infections. In contrast, we found that in the Monterey region of California, $S$. flexneri accounted for $51 \%$ of isolates, followed by $S$. sonnei at $49 \%$. Additionally, we found that $S$. flexneri types $3 \mathrm{a}$ and $1 \mathrm{~b}$ were most common, followed by $1 c, 2 a$, and a $Y$ variant $(-: 3,4)$. Subserotypes $2 \mathrm{a}$ and $3 \mathrm{a}$ have been reported as common in studies from India, Thailand, and Malaysia, while subserotype $1 \mathrm{~b}$ was the predominant type in studies from Malaysia and Peru [4]. A 2007 study [29] of adults in San Francisco, CA conducted during 1998-1999 found a similar species distribution to our study, with $S$. flexneri making up $53 \%$ of isolates and S. sonnei $46 \%$, while a previous 1996 study [27] that included all age groups found the more typical distribution of $S$. sonnei predominating over
S. flexneri at $62 \%$ versus $32 \%$, respectively. No subserotyping data was available from the San Francisco studies.

The majority of shigellosis cases in our study occurred in children. Approximately $40 \%$ of $S$. sonnei isolates were detected in children under 5 years old, with the other $60 \%$ of isolates detected in youth 5-18 years old. Shigella flexneri isolates were more evenly distributed among all age groups, with $20 \%$ of isolates occurring in children under 5 years, $40 \%$ in youth $5-18$ years, and $40 \%$ in adults over 18 years old. Most other studies have reported the majority of shigellosis cases in children $[8,11,28,30]$ though the Baer et al. study [27] in San Francisco found the majority of cases in adults over 18 years old, along with the risk factors of male gender, white ethnicity, and being HIV-positive.

Ethnicity has been associated with Shigella infection in the United States [28-31]. The Shiferaw et al. [28] study that utilized the Foodborne Diseases Active Surveillance Network (FoodNet) reported a higher incidence of $S$. sonnei and $S$. flexneri cases among patients of black and Hispanic ethnicity compared to white patients. That study also noted that the highest incidences of shigellosis in the country occurred in California and Georgia. The Kalluri et al. [31] study of $S$. boydii epidemiology reported that the majority of patients were Hispanic, and that most of the patients who had traveled abroad in the week before symptoms occurred had gone to Mexico. In our study we did not have ethnicity data linked to individual cases, but we can speculate that our results could be similarly affected given the high percentage of Hispanics with shigellosis (approximately $86 \%$ of cases from 2003-2007) in 

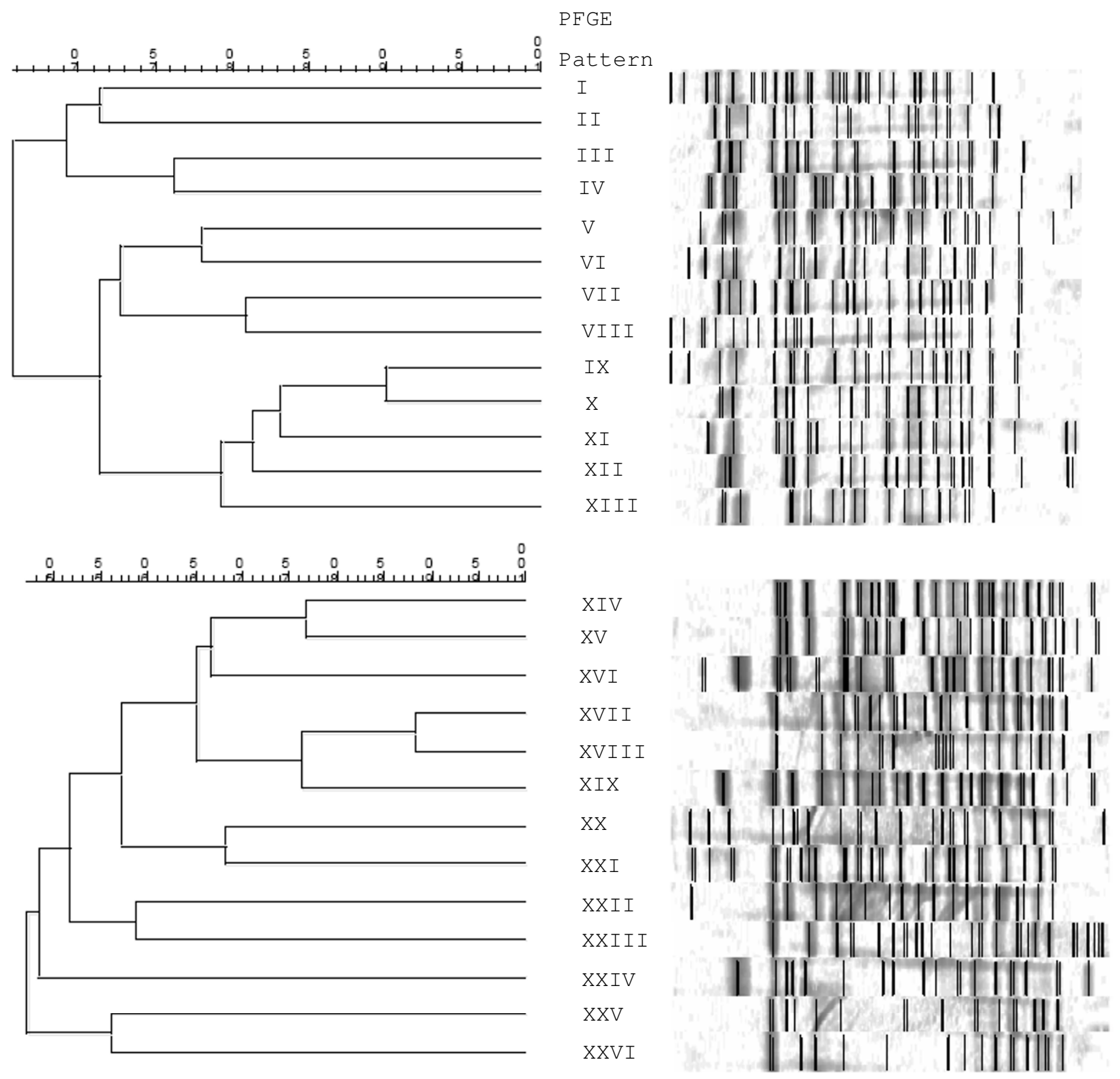

Figure 5

Shigella sonnei (top) and S. flexneri (bottom) dendrograms showing intra-species relatedness of Pulsed Field Gel Elecrophoresis (PFGE) fingerprints after Xbal digestion.

the Monterey region [32]. Many are migrant workers who travel regularly to their countries, so it is plausible that Shigella strains are being imported with these travelers. The high prevalence of $S$. flexneri could also reflect strains circulating among subgroups such as homosexual populations, as reported in San Francisco [29].

Treatment of shigellosis should be guided by local antimicrobial resistance patterns. In this central California study, we found no full resistance to amikacin, azithromycin, cefoxitime, ceftiofur, ceftriaxone, ciprofloxacin, gentamicin, and nalidixic acid. However, significant intermediate resistance was observed to azithromycin in S. sonnei isolates, and to amoxicillin-clavulanic acid in S. flexneri isolates. The results indicate that Shigella species could develop full resistance to these two drugs in the future, and this should be considered when prescribing them as empirical therapy for shigellosis. Importantly, we 
observed full resistance to many drugs commonly used for shigellosis treatment, with high resistance noted for ampicillin, chloramphenicol, streptomycin, sulfisoxazole, tetracycline, and trimethoprim-sulfa. This finding is concerning but not surprising in light of the Sur et al. [11] review that discusses how drug resistance has developed in the same order in which new drugs for shigellosis treatment were introduced. In the 1940's, tetracycline and then chloramphenicol were recommended for treatment when sulphonamides became ineffective. When further drug resistance developed, ampicillin and co-trimoxazole were recommended, and by the 1980's, some Shigella strains were resistant to all of these drugs but susceptible to the newly introduced fluoroquinolones. In recent studies, drug resistance has been observed to fluoroquinolones as well $[33,34]$.

The prevalence of resistance to individual drugs in our study was somewhat different than the NARMS data for the 2004 year. Shigella flexneri isolates in this California study showed comparable levels of resistance to the national level data, but $S$. sonnei isolates showed more resistance to streptomycin, sulfisoxazole, tetracycline, and trimethoprim-sulfa and less resistance to ampicillin than the national data. The almost $100 \%$ of S. sonnei isolates that were fully resistant to streptomycin, sulfisoxazole, tetracycline, and trimethoprim-sulfa in the California study, compared to roughly $50 \%$ of isolates found resistant in the NARMS national report, is more consistent with what you might expect in developing countries. The high level of resistance in the Monterey region could have developed in response to local use of these four antimicrobials for shigellosis treatment, or the resistant strains might have been imported from developing countries via the traveling portion of the population.

Interpretation of the disk diffusion method for azithromycin can be challenging due to the double zone of inhibition observed for many $S$. sonnei isolates. Unlike most antimicrobial disk testing, azithromycin testing of Shigella isolates can produce an inner zone of complete growth inhibition around the disk, surrounded by an outer zone of reduced growth [35]. This double zone phenomenon was observed in our study also, mainly among S.sonnei but also among a few S.flexneri isolates. In order to make an accurate and consistent interpretation of the zone diameter, it is recommended to only measure the inner zone of complete growth inhibition to interpret antimicrobial susceptibility [35]. Using the diameter of the outer zone of reduced growth to interpret antimicrobial susceptibility could result in underestimating the level of resistance.

A variety of mechanisms may be responsible for the development of antimicrobial resistance among Shigella strains. Resistance may spread clonally during an outbreak situation or horizontally by plasmids, transposonmediated conjugation, or chromosomal gene transfer [11]. In the current study we screened a subset of Shigella isolates for genes associated with antimicrobial resistance to ampicillin, chloramphenicol, and tetracycline, and found that some but not all isolates in each antimicrobial resistance category carried the target genes. Our results are consistent with the Toro et al. [5] and Navia et al. [9] studies that found a heterogeneous distribution of resistance determinants among S. flexneri and S. sonnei isolates in Chile and Tanzania, respectively. Additional molecular studies could be performed to further characterize drug resistance and virulence alleles present in study isolates.

Plasmid profiling and chromosomal fingerprinting both showed a high level of diversity among Shigella from the Monterey Bay region of California. Of the 42 isolates characterized by plasmid profiling, 62\% contained plasmids and these were distributed among ten different profiles. Of the 26 isolates characterized by PFGE, all produced unique chromosomal fingerprint patterns. The high level of diversity present in the Shigella population suggests an endemic as compared to outbreak scenario in this community, and that this suite of molecular techniques will be useful in distinguishing outbreak from endemic strains in California in the future. Indeed, other studies have used plasmid profiling and PFGE to investigate the molecular epidemiology of shigellosis in a variety of settings worldwide $[9,15,19,20]$.

\section{Conclusion}

This study showed how epidemiologic patterns at the host and bacterial population levels can be used to investigate endemic as compared to outbreak patterns of shigellosis in a community. Information gathered as part of such investigations will be instrumental in identifying emerging antimicrobial resistance, for developing treatment guidelines appropriate for that community, and to provide baseline data with which to compare outbreak strains in the future. This study confirms findings from other parts of the world that point to a continued emergence of multi-drug resistant strains of enteric pathogens in the face of widespread antimicrobial use.

\section{Competing interests}

The authors declare that they have no competing interests.

\section{Authors' contributions}

SA was involved in all parts of the study, with a main focus on the molecular characterization studies and initial manuscript preparation. WM conceived of the study, trained SA, guided the study design and analysis, and prepared the manuscript for publication. BB provided expertise and facilities for antimicrobial resistance testing, and contrib- 
uted to manuscript preparation. GG provided all isolates and contributed to manuscript preparation. LC provided expertise and facilities for the molecular characterization studies, and contributed to manuscript preparation. All authors read and approved the final manuscript.

\section{Authors' Information}

SA was a Master's student under the co-mentorship of WAM and LC. WAM is adjunct faculty at the School of Veterinary Medicine (SVM), University of California Davis (UCD) and has expertise in epidemiology and microbiology. BB is a faculty microbiologist at SVM, UCD with extensive clinical microbiology experience. GG is laboratory director at the Monterey County Public Health Department and has extensive microbiology experience. LC is a faculty microbiologist at San Francisco State University.

\section{Acknowledgements}

We thank the staff at the Monterey and Santa Cruz County Health Departments for providing the bacterial isolates and demographic data used in this study. Special thanks are extended to N. Strockbine and E. Sowers at the Centers for Disease Control and Prevention for providing subserotyping information. This study was supported in part by the NIH-NIGMS Professors of the Future Program.

\section{References}

I. Kotloff KL, Winickoff JP, Ivanoff B, Clemens JD, Swerdlow DL, Sansonetti PJ, Adak GK, Levine MM: Global burden of Shigella infections: implications for vaccine development and implementation of control strategies. Bull World Health Organ 1999, 77:65I-666.

2. Gupta A, Polyak CS, Bishop RD, Sobel J, Mintz ED: Laboratory-confirmed shigellosis in the United States, 1989-2002: epidemiologic trends and patterns. Clin Infect Dis 2004, 38: I 372-1377.

3. Levine MM, Kotloff KL, Barry EM, Pasetti MF, Sztein MB: Clinical trials of Shigella vaccines: two steps forward and one step back on a long, hard road. Nat Rev Microbiol 2007, 5:540-553.

4. Ram PK, Crump JA, Gupta SK, Miller MA, Mintz ED: Part II. Analysis of data gaps pertaining to Shigella infections in low and medium human development index countries, 1984-2005. Epidemiol Infect 2008, I36:577-603.

5. Toro CS, Farfan M, Contreras I, Flores O, Navarro N, Mora GC, Prado V: Genetic analysis of antibiotic resistance determinants in multi-drug-resistant Shigella strains isolated from Chilean children. Epidemiol Infect 2005, 133:81-86.

6. Mead PS, Slutsker L, Dietz V, McCaig LF, Bresee JS, Shapiro C, Griffin PM, Tauxe RV: Food-related illness and death in the United States. Emerg Infect Dis 1999, 5:607-625.

7. Lolekha S, Vibulbandhitkit S, Poonyarit P: Response to antimicrobial therapy for shigellosis in Thailand. Rev Infect Dis 1991, S4:S342-S346.

8. Niyogi SK: Shigellosis. J Microbiol 2005, 43:133-143.

9. Navia MM, Capitano L, Ruiz J, Vargas M, Urassa H, Schellemberg D, Gascon J, Vila J: Typing and characterization of mechanisms of resistance of Shigella spp. isolated from feces of children under 5 years of age from Ifakara, Tanzania. J Clin Microbiol 1999, 37:3||3-3|I7.

10. Peirano G, Agerso Y, Aarestrup FM, dos Prazeres Rodrigues D: Occurrence of integrons and resistance genes among sulphonamide-resistant Shigella spp. from Brazil. J Antimicrob Chemother 2005, 55:301-305.

II. Sur D, Ramamurthy T, Deen J, Bhattacharya SK: Shigellosis: challenges \& management issues. Indian J Med Res 2004, 1 20:454-462.
12. Mclver CJ, White PA, Jones LA, Karagiannis T, Harkness J, Marriott $D$, Rawlinson WD: Epidemic strains of Shigella sonnei biotype $\mathbf{g}$ carrying integrons. J ClinMicrobiol 2002, 40: I538-1540.

13. Pan JC, Ye R, Meng DM, Zhang W, Wang H, Liu KZ: Molecular characteristics of class $I$ and class 2 integrons and their relationships to antibiotic resistance in clinical isolates of Shigella sonnei and Shigella flexneri. J Antimicrob Chemother 2006, 58:288-296.

14. Pazhani GP, Sarkar B, Ramamurthy T, Bhattacharya SK, Takeda Y, Niyogi SK: Clonal multidrug-resistant Shigella dysenteriae type I strains associated with epidemic and sporadic dysenteries in eastern India. Antimicrob Agents Chemother 2004, 48:68I-684.

15. DeLappe N, O'Halloran F, Fanning S, Corbett-Feeney G, Cheasty T, Cormican M: Antimicrobial resistance and genetic diversity of Shigella sonnei isolates from western Ireland, an area of low incidence of infection. J Clin Microbiol 2003, 41:1919-1924.

16. Ahmed A, Furuta K, Shimomura K, Kasama Y, Shimamoto T: Genetic characterization of multi-drug resistance in Shigella spp. from Japan. J Med Microbiol 2006, 55: I685-169I.

17. Oh JY, Yu HS, Kim SK, Seol SY, Cho DT, Lee JC: Changes in patterns of antimicrobial susceptibility and integron carriage among Shigella sonnei isolates from southwestern Korea during epidemic periods. I Clin Microbiol 2003, 4I:42 I-423.

18. Djie-Maletz A, Reither K, Danour S, Anyidoho L, Saad E, Danikuu F, Ziniel P, Weitzel T, Wagner J, Bienzle U, Stark K, Seidu-Korko A, Mockenhaput FP, Ignatius R: High rate of resistance to locally used antibiotics among enteric bacteria from children in Northern Ghana. J Antimicrob Chemother 2008, 61:1315-1318.

19. Na-Ubol M, Samosornsuk S, von Seidlein L, Tapchaisri P, Ali M, Clemens JD, Chaicumpa W: Molecular characterization of Shigella spp. isolated from patients with diarrhoea in a new industrialized area of Thailand. Epidemiol Infect 2006, I34:997-1003.

20. Lima A, Sirim JJC, Lima NL, Titlow W, Evans ME, Greenberg RN: Molecular epidemiology of multiply antibiotic-resistant Shigella flexneri in Fortalez, Brazil. J Clin Microbiol 1997, 35:106I-1065.

21. National Committee for Clinical Laboratory Standards: Methods for dilution antimicrobial susceptibility tests for bacteria that grow aerobically 5th edition. National Committee for Clinical Laboratory Standards; 2000.

22. National Committee for Clinical Laboratory Standards: Performance standards for antimicrobial disk susceptibility tests 7 th edition. National Committee for Clinical Laboratory Standards; 2000.

23. Centers for Disease Control and Prevention: National Antimicrobial Resistance Monitoring System for enteric bacteria (NARMS): human isolates final report, 2004 United States Department of Health and Human Services; 2007.

24. Sambrook J, Fritsch EF, Maniatis T: Molecular cloning, a laboratory manual 2nd edition. Cold Spring Harbor, Cold Spring Harbor Press; 1989.

25. Centers for Disease Control and Prevention: Standardized molecular subtyping of foodborne bacterial pathogens by pulsed-field gel electrophoresis, training manual Centers for Disease Control and Prevention; 2000.

26. Pazhani GP, Niyogi SK, Singh AK, Sen B, Taneja N, Kundu M, Yamasaki $\mathrm{S}$, Ramamurthy $\mathrm{T}$ : Molecular characterization of multidrugresistant Shigella species isolated from epidemic and endemic cases of shigellosis in India. J Med Microbiol 2008, 57:856-863.

27. Baer JT, Vugia DJ, Reingold AL, Aragon T, Angulo FJ, Bradford WZ: HIV infection as a risk factor for shigellosis. Emerg Infect Dis 1999, 5:820-823.

28. Shiferaw B, Shallow S, Marcus R, Segler S, Soderlund D, Hardnett FP, van Gilder $T$ : Trends in population-based active surveillance for shigellosis and demographic variability in FoodNet sites, 1996-1999. Clin Infect Dis 2004, 38:SI75-80.

29. Aragón TJ, Vugia DJ, Shallow S, Samuel MC, Reingold A, Angulo FJ, Bradford WZ: Case-control study of shigellosis in San Francisco: the role of sexual transmission and HIV infection. Clin Infect Dis 2007, 44:327-334.

30. Naik DJ: Prevalence and antimicrobial susceptibility patterns of Shigella species in Asmara, Eritrea, northeast Africa. J Microbiol Infect 2006, 39:392-395.

31. Kalluri P, Cummings KC, Abbott S, Malcolm GB, Hutcheson K, Beall A, Joyce K, Polyak C, Woodward D, Caldeira R, Rodgers F, Mintz ED, Strockbine N: Epidemiologic features of a newly described serotype of Shigella boydii. Epidemiol Infect 2004, 132:579-583. 
32. Annual reportable diseases and conditions report, 20072008 [http://www.co.monterey.ca.us/health/Publications/CountyPublica tions.htm\#Communicable\%20Disease.]. Monterey County Health Department

33. Naheed A, Kallui P, Talukder KA, Faruqe AS, Shatun F, Nair GB, Mintz $E D$, Breimna RF: Fluoroquinolone-resistant Shigella dysentariae type I in northeastern Bangladesh. Lancet Infect Dis 2004, 4:607-608.

34. Pazhani GP, Ramamurthy T, Mitra U, Bhattacharya SK, Niyogi SK: Species diversity and antimicrobial resistance of Shigella spp. isolated between 200 I- and 2004 from hospitalized children with diarrhoea in Kolkata (Calcutta) India. Epidemiol Infect 2005, 133:1089-1095.

35. Jain SK, Gupta A, Glanz B, Dick J, Siberry GK: Antimicrobial-resistant Shigella sonnei : limited antimicrobial treatment options for children and challenges of interpreting in vitro azithromycin susceptibility. Pediatr Infect Dis J 2005, 24:494-497.

\section{Pre-publication history}

The pre-publication history for this paper can be accessed here:

http://www.biomedcentral.com/1471-2334/9/184/pre pub

Publish with Bio Med Central and every scientist can read your work free of charge

"BioMed Central will be the most significant development for disseminating the results of biomedical research in our lifetime."

Sir Paul Nurse, Cancer Research UK

Your research papers will be:

- available free of charge to the entire biomedical community

- peer reviewed and published immediately upon acceptance

- cited in PubMed and archived on PubMed Central

- yours - you keep the copyright

Submit your manuscript here:

http://www.biomedcentral.com/info/publishing_adv.asp
BioMedcentral 